\title{
SISTEMATIZACIÓN DEL PROCESO DE ORIENTACIÓN ACADÉMICA MEDIANTE MODELOS DE SELECCIÓN DE PLANES DE MATRÍCULA
}

\author{
Maureen Murillo Rivera ${ }^{1}$ \\ Vladimir LaraVillagrán ${ }^{2}$
}

\begin{abstract}
Resumen: En este artículo se propone un conjunto de modelos de selección del mejor plan de matrícula para estudiantes de la Escuela de Ciencias de la Computación e Informática de la Universidad de Costa Rica, en apoyo a la toma de decisiones durante el proceso de orientación académica. Cada modelo es una jerarquía de objetivos diseñada por medio del método "Analytic Hierarchy Process". Los modelos representan diferentes perfiles estudiantiles, especificados de acuerdo con distintos objetivos y criterios. Se enfoca el proceso de orientación académica desde el punto de vista de sistema, en donde dos agentes (estudiante y profesor) interactúan en diferentes etapas para lograr un objetivo común, la autorización de un plan de matrícula.
\end{abstract}

Descriptores: ORIENTACIÓN ACADÉMICA/ PLAN DE MATRÍCULA/ SISTEMAS DE SOPORTE A LA TOMA DE DECISIONES/ PROCESO JERÁRQUICO ANALITICO/

\begin{abstract}
We propose a set of models in order to support the decisions taken during the academic orientation process that seeks for the best set of courses that a student must attend at the Escuela de Ciencias de la Computación e Informática of the Universidad de Costa Rica. Each model represents a hierarchy of goals designed thru the "Analytic Hierarchy Process". The models correspond to different student profiles, according to different objectives and criteria. The academic orientation process is viewed as a system where two agents (student and professor) interact through several steps leading toward a common goal, the definition of an authorized set of courses to attend.
\end{abstract}

Key words: ACADEMIC GUIDANCE PROCESS/ DECISION SUPPORT SYSTEMS/ ANALYTIC HIERARCHY PROCESS/

\section{Introducción}

Tradicionalmente, la Universidad de Costa Rica (UCR), y por ende la Escuela de Ciencias de la Computación e Informática (ECCl) como unidad académica de la institución, se han preocupado por brindar una atención integral a los estudiantes (UCR, OPLAU, 1996). Las

\footnotetext{
${ }^{1}$ Magister en Computación e Informática; Licenciatura y Bachillerato en Computación e Informática, todos los títulos de la Universidad de Costa Rica. Profesora e investigadora de la Escuela de Ciencias de la Computación e Informática de la Universidad de Costa Rica.

Correo electrónico: maureenm@cariari.ucr.ac.cr

${ }^{2}$ Doctor en Informática del Instituto Polytécnico de Lorena, Francia; Licenciatura en Computación e Informática de la Universidad de Costa Rica. Actualmente es Profesor y Director de la Escuela de Ciencias de la Computación e Informática de la Universidad de Costa Rica.

Correo electrónico: vlara@ecci.ucr.ac.cr
}

Artículo recibido: 21 de abril, 2004

Aprobado: 7 de junio, 2004 
condiciones favorables que la institución les ofrezca repercuten en un mejoramiento de los porcentajes de aprobación de créditos y en un incremento en los niveles de excelencia académica. Esta excelencia ${ }^{3}$ es uno de los objetivos más importantes de la UCR como institución de educación superior. "El quehacer institucional se sustenta fundamentalmente, en la excelencia académica; por lo tanto, se deben propiciar acciones tendientes a fortalecerla" (UCR, OPLAU, 1996, p.7).

Como parte de las acciones de la UCR dirigidas a la atención del estudiante se destaca la orientación académica, la cual es llevada a cabo como etapa inicial del proceso de matrícula.

La orientación académica "es el proceso de estudio que realizan en forma conjunta el profesor consejero y el estudiante, del expediente académico que tiene la respectiva unidad, para fijar la carga académica más aconsejable para el siguiente ciclo lectivo" (UCR, 2001, cap.1, art.3, inc.n).

Como parte de este proceso de orientación la unidad académica lleva a cabo diferentes tareas, de las cuales una de las principales y de interés para este trabajo es la autorización de matrícula. Esta autorización es un proceso de toma de decisiones en donde el profesor consejero debe autorizar o rechazar los cursos que el estudiante desea matricular, luego de haber realizado un análisis detallado de la situación académica del estudiante.

El proceso de orientación académica persigue múltiples objetivos, los cuales fueron aprobados en el III Congreso Universitario (Biasetti, Gutiérrez y Quirós, 1991). El profesor consejero, por medio de la autorización que ejecuta, debe intentar que se cumplan objetivos tales como: propiciar el mejor uso de los cupos disponibles para la enseñanza, lograr que los estudiantes alcancen el máximo rendimiento posible en sus estudios y procurar que el estudiante culmine su carrera en los plazos previstos en el plan de estudios, entre otros.

La función del profesor es compleja en este proceso ya que debe considerar y analizar para cada estudiante su situación académica e incluso personal en la que se encuentra, además

\footnotetext{
${ }^{3}$ La excelencia académica en la Universidad de Costa Rica está definida por: a) un puntaje de admisión equivalente a una nota de 9.0 o superior, obtenida la primera vez que se presente la prueba de aptitud académica; b) un promedio de al menos 9.0 con una carga académica igual o superior a 15 créditos en cada ciclo lectivo correspondiente a la carrera en la cual está empadronado el estudiante (UCR, 1983).
} 
de estimar las posibles condiciones en que se desenvolverá cada uno de ellos y cómo influirán en el rendimiento académico durante el semestre siguiente. Al mismo tiempo, debe considerar las características de las diferentes opciones de planes de matrícula, es decir, las posibles combinaciones de cursos que el estudiante podría matricular.

Lo anterior en combinación con un ambiente cambiante a través del tiempo, por ejemplo en cuanto a los objetivos de la unidad académica para la matrícula, y además los objetivos personales de cada estudiante, hacen esencial la aplicación de la experiencia y criterios del profesor consejero para evaluar diferentes alternativas.

Dado que en todo este proceso de autorización de matrícula el profesor interactúa con el estudiante, es necesario promover relaciones que favorezcan la disposición del estudiante al proceso de aprendizaje (Biasetti, Gutiérrez y Quirós, 1991). La optimización de la relación profesor-estudiante es un elemento fundamental en la búsqueda de la excelencia. Debe tratarse que la atención del estudiante sea lo más individualizada posible y contenga los componentes adecuados en los ámbitos de la formación y de la orientación académica (UCR, Rectoría, OPLAU, 1985).

En este artículo se modelan situaciones típicas que contemplan estos componentes y facilitan la relación profesor-estudiante al contarse con una proposición inicial de discusión.

Los modelos aquí definidos van dirigidos a que el proceso de selección del mejor plan de matrícula para cada estudiante sea una decisión lo más racional posible, de tal forma que los criterios aplicados sean consistentes para todos los estudiantes. Al mismo tiempo, la diversidad de modelos que se definieron correspondientes a diferentes perfiles estudiantiles, permite que el proceso de decisión sea individualizado y personalizado para cada estudiante. Los modelos definidos constituyen una base para que sean modificados de acuerdo con los criterios personales de los tomadores de decisiones y de las particularidades de cada estudiante.

El desarrollo de los modelos propuestos está basado en las particularidades y necesidades actuales de la ECCI como unidad académica de la UCR. 


\section{Procesos de análisis y decisión en la orientación académica}

Como se mencionó, la orientación académica está definida en el Reglamento de Régimen Académico Estudiantil como el proceso de análisis del expediente del estudiante para definir la carga académica más aconsejable. Es común encontrar una descripción más amplia de este proceso en términos de acciones independientes que deben y pueden llevar a cabo el profesor y el estudiante. Por ejemplo, asignación de profesor consejero, fórmulas que deben llenarse, funciones del profesor y derechos del estudiante, como por ejemplo, cambiar de profesor consejero (UCR, 2001).

Sin embargo, el proceso de orientación académica puede ser mucho más complejo de lo que generalmente se describe, en situaciones en que tanto el estudiante como el profesor desean aprovechar al máximo la orientación. Desde el momento en que el estudiante y su profesor guía se reúnen hasta que el plan de matrícula es autorizado, ambos interactúan continuamente.

De esta forma, la orientación académica se convierte en un sistema, entendido este término como un conjunto de componentes que interactúan entre sí para lograr un propósito (Senn, 1987). En el proceso de orientación dos personas con inteligencia y criterios propios interactúan, analizan y toman decisiones para tratar de llegar a un acuerdo sobre la carga académica más recomendable.

Ante la pregunta de un profesor que llevará a cabo la orientación académica por primera vez sobre cuáles son los pasos a seguir con el estudiante para alcanzar el resultado deseado, el enfoque de sistema dado al proceso de orientación resulta de gran utilidad.

En este artículo se presenta la orientación académica como un sistema con procesos de análisis y toma de decisiones, relacionados entre sí por medio de entradas y salidas. Estos procesos se muestran esquemáticamente en la figura 1 y se explican a continuación: 


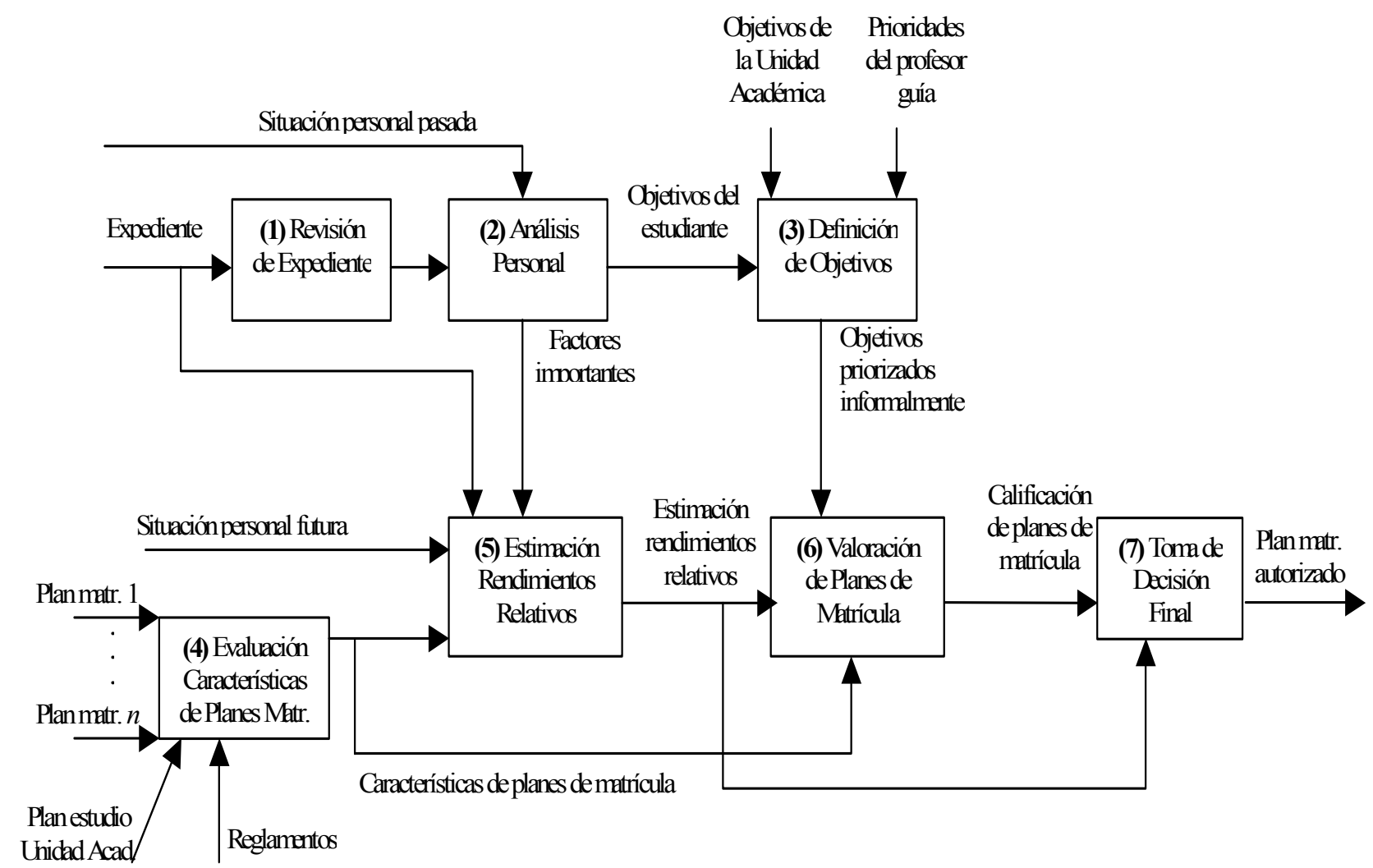

Figura 1: Procesos de Análisis y Decisión en la Orientación Académica

1) Revisión del expediente académico del estudiante: el profesor consejero revisa el rendimiento académico histórico del estudiante y los resultados obtenidos en el semestre recién finalizado.

2) Análisis personal: conociendo el expediente, el profesor analiza en conjunto con el estudiante su situación personal que afectó directa o indirectamente su desempeño académico. De este análisis se determina cuáles factores son los que influyen en su rendimiento para ser tomados en cuenta para el semestre siguiente y los objetivos que el estudiante tiene con respecto a su plan de estudio.

3) Definición de objetivos: tomando en cuenta los objetivos planteados por el estudiante, los definidos por la Unidad Académica y las prioridades que el profesor guía establezca, el estudiante y el profesor deben definir la lista de preferencias de los objetivos que se pretenden alcanzar. 
4) Evaluación de las características de los planes de matrícula: dadas varias alternativas de planes de matrícula (diferentes combinaciones y agrupamientos de cursos), se analizan sus características, por ejemplo, dificultad promedio de los cursos, número total de créditos, semestre de los cursos con respecto a la ubicación dentro del plan de estudio de la carrera, tipo de profesores que impartirán los cursos, cumplimiento con lo establecido por los reglamentos y directrices de la UCR y de la Unidad Académica, entre otros.

5) Estimación de rendimientos relativos: el rendimiento académico que el estudiante podría tener con cada una de las alternativas de matrícula es, entre otras, una de las variables que debe tomarse en cuenta para seleccionar el mejor plan. Estas estimaciones no necesariamente deben obtenerse en la misma escala del promedio ponderado, simplemente puede utilizarse una medida relativa a cada uno de los planes de matrícula que permita clasificarlos según el posible rendimiento académico. Esta estimación está basada generalmente en la experiencia del profesor guía, el cual analiza las características de los diferentes planes de matrícula, los factores que influyen en el rendimiento académico de cada estudiante y la situación personal que tendrá en el siguiente semestre. Para controlar parcialmente la subjetividad propia de cada profesor pueden establecerse mecanismos de cálculo de los rendimientos relativos. Por ejemplo, pueden priorizarse mediante pesos las características de los planes de matrícula y los factores que influirán en el rendimiento del estudiante, asignándoles luego un valor según cada plan de matrícula, que será multiplicado por el peso. De esta forma se obtendría una medida de la bondad de cada uno de los planes. Puede emplearse también algún modelo formal de estimación, tal como el propuesto en (Murillo y Lara, 2002).

6) Valoración de planes de matrícula: en este proceso el profesor consejero y el estudiante toman directa o indirectamente los resultados obtenidos de los procesos previos, los analizan y concilian todos los elementos para valorar y darle un puntaje o calificación a cada plan de matrícula. Esta valoración se realiza tomando en cuenta las características de cada plan y los rendimientos estimados, tratando de establecer su adecuación con respecto a los objetivos definidos previamente.

7) Toma de decisión final: dada la calificación de los planes de matrícula, la última etapa es la selección final del plan a autorizar. La calificación obtenida en el proceso anterior sugiere autorizar el plan con mayor puntaje. Los tomadores de decisiones (el estudiante y el profesor 
guía) en esta etapa validan la calificación dada por el proceso anterior. Esta validación va desde la verificación de los resultados con respecto al historial académico del estudiante, hasta el eventual levantamiento de ciertos requisitos que ubicaban a un plan de matrícula particular en una posición baja de la clasificación.

\section{Modelaje}

Los modelos de clasificación de alternativas que se proponen en este artículo, apoyan la toma de decisión de selección del plan de matrícula más adecuado para el estudiante, definiendo varios modelos por perfiles de estudiantes. Estos modelos representan uno de los procesos definidos anteriormente, el de valoración de planes de matrícula, en donde se toman los resultados de los procesos anteriores y se conjugan para obtener una calificación de los planes de matrícula. La combinación de los diferentes objetivos y criterios no es inmediata y en algunos casos se contraponen. Por ejemplo, algunos estudiantes buscan mejorar su rendimiento académico al mismo tiempo que avanzar rápido en la carrera aprobando la mayor cantidad de créditos. El matricular un elevado número de créditos puede afectar negativamente el rendimiento, o visto de otra forma, disminuir los créditos puede influir positivamente en el rendimiento académico. Debido a esta dificultad de conciliación de objetivos se le dio al problema un enfoque de toma de decisiones con objetivos múltiples.

\subsection{Toma de decisiones con objetivos múltiples}

La mayoría de las decisiones están asociadas a conflictos. Estos conflictos se dan por la presencia de múltiples objetivos, los cuales a veces compiten entre sí. En algunos otros casos no se presenta esta contraposición pero permanece la multiplicidad de objetivos, los cuales es necesario combinar para tomar una decisión. En el caso de la autorización de la carga académica de los estudiantes existe diversidad de objetivos en la toma de decisiones e incluso los objetivos de la Unidad Académica pueden entrar en conflicto con los de los estudiantes.

Los métodos disponibles para resolver problemas con objetivos múltiples provienen de áreas de estudio diferentes. En Fandel y Spronk, 1985) se propone la siguiente clasificación para estas áreas: análisis de datos y escalas multidimensionales, investigación operacional y 
programación matemática, teoría de utilidad, teoría del bienestar social y teoría de medición psicosensitiva.

El método Analytic Hierarchy Process (AHP) ha probado ser muy útil en problemas de toma de decisiones que involucran una selección de múltiples objetivos (Olson y Courtney, 1992). Este fue el método utilizado para el diseño de los modelos propuestos en este artículo, cada uno de los cuales constituye una jerarquía de objetivos y criterios. A continuación se explica el método AHP.

\subsection{Método "Analytic Hierarchy Process" (AHP)}

El método AHP fue desarrollado por Thomas L. Saaty en la década de los setentas. Ha sido utilizado en el desarrollo de funciones objetivo combinadas con pesos representando una serie de objetivos en aplicaciones de programación matemática. AHP provee un medio para convertir criterios subjetivos de relativa importancia en un conjunto lineal de pesos, los cuales pueden usarse para clasificar alternativas o para servir como una función objetivo en otras técnicas (Olson y Courtney, 1992).

La aplicación del AHP a un problema particular se realiza en tres pasos generales:

1) Descomposición del problema: Los problemas se descomponen produciendo una jerarquía de factores objetivo o criterios. Generalmente se inicia definiendo la meta de la solución, que en este caso es la selección de un plan de matrícula. Luego se especifican los objetivos de la decisión y, si es necesario, otros niveles con subobjetivos. Finalmente en el último nivel se especifican los factores o criterios con los cuales serán evaluadas las alternativas de decisión.

2) Juicios comparativos: Como segundo paso, se identifica la importancia relativa de los nodos de la jerarquía comparando de dos en dos la importancia proporcional de los criterios de una misma rama y nivel. Saaty propone la siguiente escala comparativa (Olson y Courtney, 1992): 


\begin{tabular}{|c|l|}
\hline Escala & $\begin{array}{c}\text { Relación del factor base con } \\
\text { respecto al otro factor }\end{array}$ \\
\hline 1 & Igualmente importante \\
\hline 3 & Moderadamente más importante \\
\hline 5 & Fuertemente más importante \\
\hline 7 & Muy fuertemente más importante \\
\hline 9 & Extremadamente más importante \\
\hline
\end{tabular}

A partir de las comparaciones realizadas, debe obtenerse el peso general para cada elemento de la jerarquía. Existen dos formas principales para la obtención de estos pesos, ya sea realizando una aproximación manual o mediante el vector propio (eigenvector) ${ }^{4}$ de las matrices de comparación propuesto por Saaty (Olson y Courtney, 1992).

En la aproximación manual primero se normalizan las columnas, dividiendo cada elemento de una columna entre la suma de todos los elementos de la columna. Luego se promedian los valores normalizados de cada fila. Los valores resultantes corresponden a los pesos de cada nodo de la jerarquía (Mazur, 1998).

El mecanismo del vector propio es más complejo y generalmente se utiliza algún programa computacional para realizar los cálculos. Con este método es posible obtener los índices de inconsistencia respectivos, los cuales son una medida de la falta de consistencia en un conjunto de comparaciones pareadas (Forman, 1990).

3) Síntesis: Con los pesos obtenidos en la etapa anterior se construye la función objetivo. Luego se utiliza esta función para evaluar cada alternativa, asignándole a cada variable (criterios en el último nivel de la jerarquía) un valor de acuerdo con alguna escala definida previamente, por ejemplo, una escala con valores entre 0 y 100.

Siguiendo los tres pasos mencionados, el AHP incorpora tanto los aspectos cualitativos como los cuantitativos del pensamiento humano: el cualitativo para definir el problema y su

\footnotetext{
${ }^{4}$ Un vector propio es un vector, el cual cuando se le realiza una transformación lineal particular, produce un múltiplo escalar del vector original. Este escalar es llamado el valor propio (eigenvalor) correspondiente a este vector propio (University of London, 1996).
} 
jerarquía, y el cuantitativo para expresar juicios y preferencias en forma concisa (Saaty, 1996).

El AHP muestra cómo conectar elementos de una parte del problema con aquellos correspondientes a otra parte para obtener una salida combinada. Es un proceso para identificar, entender y evaluar las interacciones de un sistema como un todo.

Al tratar de modelar con el AHP la solución de apoyo a la toma de decisión sobre el mejor plan de matrícula para estudiantes, se encontró que no es posible desarrollar un único modelo que convenga a toda la población estudiantil, dada la variedad de objetivos presentes. Por lo tanto, como solución se optó por identificar algunos perfiles estudiantiles y definir un modelo, utilizando el AHP, para cada uno de éstos. La definición de estos perfiles se presenta a continuación.

\subsection{Perfiles de estudiantes}

En el momento de seleccionar el mejor plan de matrícula para un estudiante, no existe un modelo único de apoyo a esta decisión que dé buenos resultados y que sea aplicable a todos los estudiantes. Esto debido a que cada persona tiene prioridades bien definidas en cuanto a los objetivos académicos que persigue y además tiene necesidades particulares según su situación académica y personal. Al mismo tiempo, deben considerarse los lineamientos de la UCR que aplican en cada caso particular.

Sin embargo, sí podrían definirse tipos de estudiantes, que sin perder su individualidad, permiten clasificar a los alumnos según objetivos y características similares. Esta clasificación por perfiles favorece un trato equitativo para los estudiantes que se encuentran en una situación similar y a la vez facilita el proceso de toma de decisión al ofrecer un esquema inicial de trabajo. Por esta razón, se definieron varios perfiles estudiantiles, obedeciendo a objetivos y características de índole académico, cada uno con su correspondiente modelo de selección de un plan de matrícula.

El Reglamento de Régimen Académico Estudiantil identifica algunos tipos de estudiante, basándose en su promedio ponderado y en su carga académica. Por ejemplo, define al 
estudiante de tiempo completo como aquel que lleva una carga de 16 créditos o más por ciclo lectivo ordinario (UCR, 2001, cap.1, art.3, inc.d).

También define tres tipos de estudiante para clasificar a los que tienen un rendimiento académico bajo (inferior a 7.0): a) estudiante en condición académica de atención especial; b) estudiante con matrícula restringida, y c) estudiante con matrícula suspendida (UCR, 2001, cap.1, art.3, inc. $r$ y y).

En el Reglamento, la definición de estos tres tipos de estudiante con bajo rendimiento va asociada con medidas de atención especial y regulaciones para la matrícula. Sin embargo, para definir los perfiles que permitan una buena toma de decisión en cuanto al mejor plan de matrícula deben considerarse otros aspectos, tales como prioridades en los objetivos académicos y ciertos elementos de apoyo para que el estudiante pueda avanzar satisfactoriamente, por ejemplo, gusto por los cursos que matriculará. Además, deben identificarse perfiles de otros tipos de estudiantes que no necesariamente tienen rendimiento académico bajo.

Teniendo en cuenta los tipos de estudiantes más frecuentes que asisten al proceso de orientación académica según la experiencia de los autores, y como propuesta inicial de un conjunto base, se definen los siguientes perfiles estudiantiles:

1. Perfil de excelencia académica: este perfil define a los estudiantes de tiempo completo que buscan mantener un alto rendimiento académico, generalmente matriculando cursos de la carrera en la que están empadronados. Dentro de estos estudiantes pueden mencionarse aquellos que: acostumbran tener notas de 9 o más, desean obtener una beca para estudios de posgrado, desean trasladarse de carrera mediante excelencia académica y quienes desean mantener una beca de excelencia o graduación de honor.

2. Perfil de avance ordenado: define a los estudiantes que quieren avanzar en su carrera según la estructura de bloques y en el plazo previsto por su respectivo plan de estudio. En este perfil se pueden ubicar a dos tipos de estudiantes: los que vienen avanzando ordenadamente y quieren mantener esa característica, y los que tienen cursos perdidos y rezagados y quieren completar bloques incompletos de cursos para luego seguir avanzando ordenadamente. 
3. Perfil de pronta graduación: este perfil hace referencia a aquellos estudiantes que buscan terminar con su plan de estudio y graduarse lo más rápido posible. Generalmente tratan de llevar la mayor cantidad de créditos que el profesor autorice, aún bajo el riesgo de perder algún curso.

4. Perfil de avance lento: en este perfil se incluyen diversos tipos de estudiantes, que por diferentes razones desean matricular pocos créditos. Se pueden mencionar, por ejemplo: los estudiantes que disponen de poco tiempo para el estudio, ya sea porque trabajan, porque tienen responsabilidades familiares o situaciones especiales de salud. También están los estudiantes que carecen de motivación para graduarse o estudiar, debido a que éste no es el objetivo primordial al asistir a la universidad, o porque no se encuentran en la carrera deseada, o bien por la frustración que sufren por su condición de bajo rendimiento. Por otro lado, se puede mencionar a los estudiantes con una situación económica limitada y que no poseen beca a causa del bajo rendimiento, por lo cual se ven obligados a matricular pocos créditos porque no pueden cancelar el costo. Por último, dentro de este perfil pueden ubicarse también los estudiantes que interrumpen sus estudios momentáneamente y luego se reincorporan, generalmente, en forma paulatina.

5. Perfil de bajo rendimiento: define a los estudiantes que tienen un promedio ponderado inferior a 7.0, incluyendo a los que se encuentran en condición académica de atención especial y los de matrícula restringida. No se incluyen los estudiantes con matrícula suspendida ya que según el Reglamento de Régimen Académico Estudiantil éstos no pueden matricularse en ningún curso durante dos años lectivos la primera vez y cuatro años lectivos cada vez que reincida (UCR, 2001, cap.8, art.41).

6. Perfil de formación académica amplia: este perfil corresponde a los estudiantes que desean recibir formación académica universitaria de carácter interdisciplinario, generalmente de distintos campos o áreas de estudio. Entre estos estudiantes están los que tratan de obtener la mayor cantidad de conocimiento posible matriculando diversidad de cursos, los que cursan dos carreras o los que asisten a dos universidades.

Esta definición de perfiles cubre los tipos de estudiantes más frecuentes de la ECCI según la experiencia de los autores. Estos perfiles fueron validados, además, por la M.Sc. Patricia 
Ruh, jefe del Centro de Orientación Vocacional de la Oficina de Orientación de la UCR. Podrían definirse otros, tal como los sugeridos por la señora Ruh: el perfil del estudiante de zonas alejadas al centro universitario, cuyo objetivo es la conveniencia de horarios de los cursos a matricular, y un perfil cuyo objetivo sea seleccionar el plan de matrícula con la probabilidad más alta de ser asignado por el sistema de matrícula de la universidad.

\section{Modelos}

Para cada uno de los perfiles mencionados anteriormente, se definió un modelo cuyo objetivo principal es seleccionar el mejor plan de matrícula para un estudiante particular. Cada modelo es una jerarquía de objetivos y criterios con sus pesos respectivos, en donde las hojas del árbol son las características que deben evaluarse para cada plan de matrícula. Los pesos ${ }^{5}$ del árbol representan la importancia relativa de cada nodo con respecto a los otros del mismo nivel en la toma de decisión que se está llevando a cabo ${ }^{6}$.

Antes de utilizar los modelos, el profesor consejero debe seleccionar el perfil que mejor represente al estudiante para así usar el modelo respectivo. Sin embargo, es claro que dada la individualidad de cada persona es posible que este modelo no sea completamente fiel a las características y prioridades del estudiante y del profesor que lo orienta. Por esta razón, los modelos que se proponen son modelos base que pueden ser modificados en su estructura y pesos según sea necesario.

Para cada modelo se obtuvo el índice de inconsistencia, el cual mide la falta de consistencia en el conjunto de comparaciones pareadas que sirvieron de base para obtener los pesos del árbol. Saaty ha propuesto como límite para el índice de inconsistencia un valor de 0.10 , es decir, 10\% (Forman, 1990). Si se obtiene un índice mayor, las comparaciones deberían ser revisadas nuevamente.

A continuación se describen los modelos, presentando para cada uno una figura con la jerarquía y los pesos de cada nodo. Además, se explica la jerarquía describiendo el objetivo

\footnotetext{
${ }^{5}$ Las prioridades que sirvieron de base para obtener los pesos de los nodos del árbol son resultado de la experiencia de los autores como profesores de la Escuela de Ciencias de la Computación e Informática. Estos pesos son una propuesta y pueden ser modificados según otras preferencias.

${ }^{6}$ Los modelos, incluyendo su jerarquía y pesos, fueron validados por la M.Sc. Patricia Ruh, jefe del Centro de Orientación Vocacional de la Oficina de Orientación de la UCR. Además, fueron evaluados por María Elena Rodríguez, psicóloga del Centro de Asesoría Estudiantil (CASE) de Ingeniería (Ilamado anteriormente Vida Estudiantil) de la UCR.
} 
principal, los subobjetivos y los criterios, con los correspondientes índices de inconsistencia. Por último se presenta la función objetivo que resulta de los pesos y la jerarquía de los modelos.

\subsection{Modelo de excelencia académica (figura 2)}

El propósito de este modelo es la selección del plan de matrícula que de la mejor forma maximice la excelencia académica en términos de rendimiento académico de un estudiante. La definición de excelencia académica en la que se basa este modelo es la adoptada oficialmente por la UCR: a) un puntaje de admisión equivalente a una nota de 9.0 o superior, obtenida la primera vez que se presente la prueba de aptitud académica; b) un promedio de al menos 9.0 con una carga académica igual o superior a 15 créditos en cada ciclo lectivo correspondiente a la carrera en la cual está empadronado el estudiante (UCR, 1987).

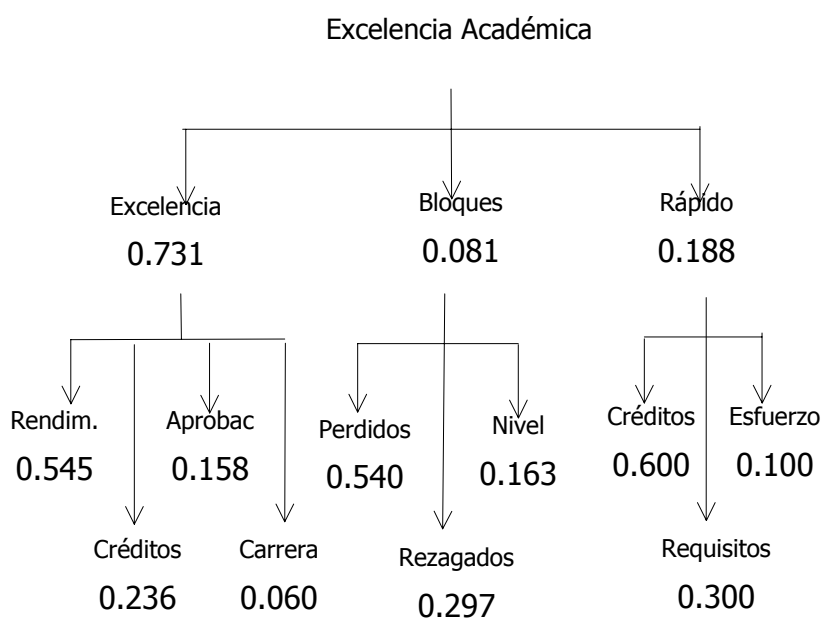

Figura 2: Modelo de excelencia académica

\subsubsection{Objetivos}

Dado el propósito de este modelo, el objetivo más importante es la excelencia académica en la carrera. Además, el modelo incluye otros dos objetivos.

El objetivo de avanzar rápido pretende que el estudiante se gradúe en el menor tiempo posible, incluso en un período de tiempo menor que el establecido por el plan de estudio. Este objetivo es el segundo en importancia debido a que se ha observado que los 
estudiantes que buscan la excelencia por lo general tienen metas altas, tal como terminar su carrera en menos del tiempo establecido por el plan de estudio.

El objetivo de completar bloques es el tercero en importancia dentro del modelo y consiste en aprobar los cursos por bloques según lo propuesto por el plan de estudio, es decir, avanzar ordenadamente. Su relevancia radica en que es deseable que un estudiante no deje rezagados cursos o que no matricule un curso de un bloque muy avanzado con respecto a su nivel actual.

Las comparaciones pareadas de estos tres objetivos dieron como resultado sus respectivos pesos mostrados en la figura 2, con un índice de inconsistencia de 0.06.

\subsubsection{Criterios}

Cada uno de los objetivos está compuesto por una serie de criterios relacionados con los planes de matrícula que conllevan a la consecución de los primeros. A continuación se presentan los criterios que conforman cada objetivo, enumerados en orden de prioridad.

Objetivo de excelencia académica en la carrera (con un índice de inconsistencia de 0.05):

1. Estimación del rendimiento académico: corresponde al posible rendimiento que tendría el estudiante si cursa determinado plan de matrícula. Esta estimación la puede realizar el profesor con base en su experiencia, aplicar algún modelo formal de estimación o puede utilizarse como base el promedio ponderado. Este criterio es el más importante de todos dentro del objetivo de excelencia académica en la carrera, ya que considerando los dos factores tomados en cuenta en la definición de excelencia académica de la UCR (promedio ponderado y cantidad de créditos), el promedio ponderado es el criterio principal en el que se basa la institución para la asignación de diversos beneficios (cupos de matrícula y becas, entre otros) y el que determina la caracterización de un estudiante como de buen rendimiento.

2. Número de créditos: corresponde a la cantidad de créditos de un plan de matrícula. Este criterio es el segundo en importancia ya que es el otro elemento en el que se basa la definición de excelencia académica de la UCR. Un plan con 15 o más créditos obtiene la calificación máxima en este criterio. 
3. Probabilidad de aprobar todos los cursos del plan de matrícula: relacionado con la excelencia académica, existen otros conceptos tal como la graduación de honor, la cual "se otorga tomando en cuenta todas las asignaturas del plan de estudios de la carrera (aún las básicas), con un promedio ponderado no inferior a 9 y sin haber perdido ningún curso" (UCR, VVE, ORI, 2002, anexo). De esta definición se destaca que es importante no perder ningún curso, por lo que al analizar un plan de matrícula debe tomarse en cuenta la probabilidad de que el estudiante apruebe todos los cursos que matriculará. Este criterio está relacionado con el posible rendimiento académico que tendrá, porque entre mayor sean el rendimiento o promedio ponderado, mayor es la probabilidad de aprobar todos los cursos. Sin embargo, debe considerarse por separado, ya que cierta combinación de cursos podría reflejarse en un promedio relativamente alto aún con una probabilidad alta de reprobar algún curso específico. Por ejemplo, un plan con tres cursos obteniendo notas de 9.5, 9.5 y 6.5, lo que da un promedio de 8.5. Por otro lado, puede presentarse la situación de que el estudiante con otro grupo de 3 cursos tendría un rendimiento promedio no tan bueno de 7.5 , con una probabilidad alta de no perder ningún curso.

4. Cursos de carrera: tal como se mencionó en el criterio anterior y como lo establece el Reglamento de los Actos de Graduación (UCR, 1983, art.7), la graduación de honor toma en cuenta sólo los cursos de la carrera, por lo es preferible que los criterios anteriores se den en conjunto con la matrícula de este tipo de cursos. Además, el modelo de excelencia académica está dirigido a los estudiantes que enfocan sus esfuerzos en culminar sus estudios en un plan de estudios particular. Un plan de matrícula en donde todos los cursos son de la carrera en la que está empadronado el estudiante obtiene la calificación máxima en este criterio.

- Objetivo de avanzar rápido en la carrera (con un índice de inconsistencia de 0.00):

1. Número de créditos: generalmente se asocia el avanzar rápido con matricular muchos créditos, aunque deben tomarse en cuenta otros factores como el aprobarlos. Sin embargo, la aprobación es un criterio que se toma en cuenta en el objetivo anterior. Entre más créditos tenga el plan de matrícula mayor es la calificación en este criterio. Por ejemplo, un plan con una calificación aceptable en este criterio debería incluir al menos la cantidad de créditos que corresponde a un bloque completo del plan de estudio. A pesar de que el número de créditos ya fue considerado en el objetivo de excelencia académica, la evaluación de este criterio es diferente en las dos ramas del árbol. Por ejemplo, un plan con 15 créditos alcanza 
la máxima calificación en el criterio de número de créditos del objetivo de excelencia académica en la carrera, mientras que en el objetivo de avanzar rápido obtendría una calificación baja si un bloque completo del plan de estudio está formado de 20 créditos.

2. Porcentaje de cursos requisito: además de un número elevado de créditos, es importante seleccionar el tipo de cursos a matricular. Una de las limitaciones con las que se topa un estudiante para matricular un curso son los requisitos que posee. Dado que el alumno tratará de matricular un número elevado de créditos en cada semestre, el aprobar lo antes posible los cursos requisito facilita las matrículas posteriores. Si un plan de matrícula incluye todos los posibles cursos requisito que el alumno puede matricular, entonces el plan obtiene la calificación máxima en este criterio.

3. Esfuerzo: consiste en la estimación del esfuerzo promedio que el alumno deberá invertir para cursar y aprobar todos los cursos. Entre menor esfuerzo requieran los cursos del plan de matrícula, mayor es la calificación en este criterio. Por ejemplo, el estudiante puede aplicar este criterio en la escogencia entre dos grupos de un mismo curso con profesores diferentes. Este criterio es de importancia en el objetivo de avanzar rápido, ya que al matricular gran cantidad de créditos debe tratar de minimizar el esfuerzo que le requerirá cada uno de los cursos para aumentar la probabilidad de aprobarlos todos.

- Objetivo de completar bloques del plan de estudio (con un índice de inconsistencia de $0.01)$ :

1. Porcentaje de cursos perdidos: los cursos perdidos por el estudiante son un componente para completar los bloques del plan de estudio. Este criterio es el primero en importancia ya que los cursos perdidos se convierten en cursos que en potencia podrían irse quedando rezagados con respecto al plan de estudio. Un plan de matrícula que incluya todos los cursos que el estudiante haya perdido obtiene una calificación máxima en este criterio.

2. Porcentaje de cursos rezagados: este criterio corresponde a los cursos que el estudiante tenga atrasados según el plan de estudio y que nunca ha matriculado. Es el segundo en importancia ya que antes de matricular cursos del nivel correspondiente del plan, deben completarse bloques previos del plan. Un plan de matrícula que incluya todos los cursos que el estudiante tenga rezagados obtiene una calificación máxima en este criterio. 
3. Porcentaje de cursos del nivel: corresponde a los cursos del nivel o bloque que el estudiante se encuentra cursando según el plan de estudio. Un plan de matrícula que incluya todos los cursos del nivel, es decir, el bloque completo, obtiene una calificación máxima en este criterio.

La organización jerárquica de todos los objetivos y criterios del modelo de excelencia académica junto con sus pesos se muestra en la figura 2.

\subsubsection{Función objetivo:}

$$
\begin{aligned}
& 0.731^{*}\left(0.545^{\star} \text { Rend }+0.236^{*} \text { Créd }+0.158^{*} \text { Aprob }+0.060^{*} \text { Carr }\right)+ \\
& 0.18 *^{*}\left(0.600^{*} \text { Créd }+0.300^{*} \text { Requis }+0.100^{*} \text { Esfuerzo }\right)+ \\
& 0.081^{*}\left(0.540^{\star} \text { Perd }+0.297^{*} \text { Rezag }+0.163^{\star} \text { Nivela }\right)
\end{aligned}
$$

Este modelo, al igual que los otros, puede ser modificado para adaptarse a diferentes criterios. Por ejemplo, en el modelo de excelencia académica propuesto el objetivo de avanzar rápido es más importante que el de avanzar por bloques. Sin embargo, otros tomadores de decisiones podrían considerar más importante el avance por bloques. Otro ejemplo de una posible variación al modelo es modificar los pesos de los criterios del objetivo excelencia académica en la carrera, al considerarse que es más importante el aprobar todos los cursos que el rendimiento académico en sí o el número de créditos matriculados.

\subsection{Modelo de avance ordenado (figura 3)}

El propósito de este modelo es la selección del mejor plan de matrícula que le permita al estudiante avanzar en el plan de estudio ordenadamente, según los bloques de cursos establecidos por el plan y, de ser posible, graduarse en el tiempo fijado.

\subsubsection{Objetivos}

En este modelo, el objetivo principal es avanzar en el plan de estudio en forma estructurada, por bloques, y de ser posible con buen rendimiento. La presencia en el modelo del objetivo de avanzar rápido, cuya prioridad es la más baja, responde a una expectativa menor que el 
estudiante muchas veces tiene de graduarse lo más pronto posible, aunque tenga claro que lo principal como mínimo es avanzar ordenadamente.

Las descripciones de estos objetivos son iguales a las del modelo de excelencia académica. En lo que se diferencian es en los pesos que les fueron asignados y en el índice de inconsistencia al comparar los objetivos, el cual es de 0.06 .

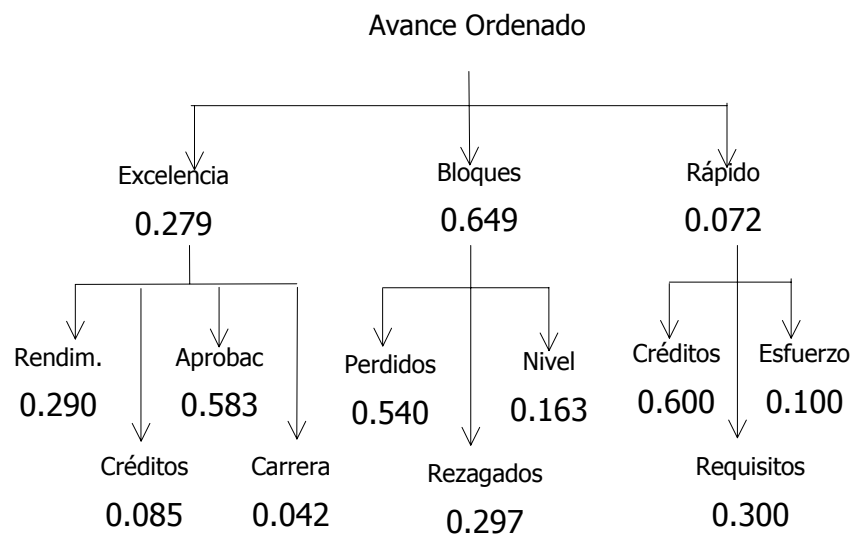

Figura 3: Modelo de avance ordenado

\subsubsection{Criterios}

Los criterios que conforman cada objetivo son los mismos que se explicaron en el modelo anterior. La variación se da en los pesos asignados y en el índice de inconsistencia obtenido. A continuación se enumeran en orden de prioridad los criterios de cada objetivo.

- Objetivo de completar bloques del plan de estudio (con un índice de inconsistencia de $0.01)$ :

1. Porcentaje de cursos perdidos.

2. Porcentaje de cursos rezagados.

3. Porcentaje de cursos del nivel.

Objetivo de excelencia académica en la carrera (con un índice de inconsistencia de 0.06):

1. Probabilidad de aprobar todos los cursos del plan de matrícula.

2. Estimación del rendimiento académico

3. Número de créditos. 
4. Cursos de carrera.

Objetivo de avanzar rápido en la carrera (con un índice de inconsistencia de 0.00):

1. Número de créditos.

2. Porcentaje de cursos requisito.

3. Esfuerzo.

La organización jerárquica de estos objetivos y criterios se muestra en la figura 3.

\subsubsection{Función objetivo:}

$0.649 *\left(0.540^{*}\right.$ Perd $+0.297^{*}$ Rezag $+0.163^{*}$ Nivela $)+$

$0.279 *\left(0.290^{*}\right.$ Rend $+0.085^{\star}$ Créd $+0.583^{*}$ Aprob $+0.042^{*}$ Carr $)+$

$0.072 *\left(0.600^{*}\right.$ Créd $+0.300 *$ Requis $+0.100 *$ Esfuerzo $)$

\subsection{Modelo de pronta graduación (figura 4)}

El objetivo principal de este modelo es seleccionar el plan de matrícula que mejor favorezca el rápido avance del estudiante en su plan de estudio, de ser posible para que se gradúe en menos del tiempo establecido por el plan.

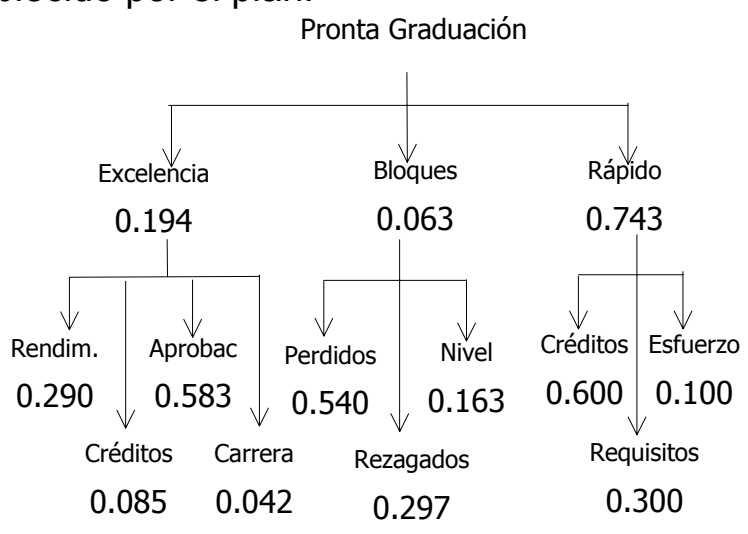

Figura 4: Modelo de pronta graduación

\subsubsection{Objetivos}

Dado el propósito de este modelo, de los tres objetivos el más importante es avanzar rápido. La excelencia académica en la carrera se convierte en el objetivo con segunda prioridad, 
para tratar de maximizar el rendimiento académico, y por último se incluye el objetivo de completar bloques. La descripción de estos tres objetivos es igual a la del modelo de excelencia académica. De su comparación pareada se obtuvo un índice de inconsistencia de 0.07 .

\subsubsection{Criterios}

La descripción de los criterios de cada objetivo son los mismos de los modelos anteriores.

Objetivo de avanzar rápido en la carrera (con un índice de inconsistencia de 0.00):

1. Número de créditos.

2. Porcentaje de cursos requisito.

3. Esfuerzo.

- Objetivo de excelencia académica en la carrera (con un índice de inconsistencia de 0.06):

1. Probabilidad de aprobar todos los cursos del plan de matrícula.

2. Estimación del rendimiento académico

3. Número de créditos.

4. Cursos de carrera.

- Objetivo de completar bloques del plan de estudio (con un índice de inconsistencia de $0.01)$ :

1. Porcentaje de cursos perdidos.

2. Porcentaje de cursos rezagados.

3. Porcentaje de cursos del nivel.

La jerarquía de objetivos y criterios de este modelo se muestra en la figura 4.

\subsubsection{Función objetivo:}

$0.743 *(0.600 *$ Créd $+0.300 *$ Requis $+0.100 *$ Esfuerzo $)+$ 


\section{$0.194^{*}\left(0.290^{*}\right.$ Rend $+0.085^{*}$ Créd $+0.583^{*}$ Aprob $+0.042^{*}$ Carr $)+$ 0.063 * $\left(0.540^{*}\right.$ Perd $+0.297^{*}$ Rezag $+0.163^{*}$ Nivela $)$}

\subsection{Modelo de avance lento (figura 5)}

El objetivo principal de este modelo es seleccionar el plan de matrícula que mejor se ajuste a las necesidades del estudiante de llevar pocos créditos, tratando de no desatender completamente algunos criterios de excelencia y rendimiento académico. Además, se procura que no deje rezagados cursos.

\subsubsection{Objetivos}

Los objetivos principales son: permanencia en la carrera, completar bloques del plan de estudio y excelencia académica en la carrera. Un estudiante que avanza lento en su carrera posiblemente esté involucrado en otras actividades (familiares, laborales, etc.), que podrían llevar al estudiante a abandonar sus estudios. Por esta razón, el objetivo principal de este modelo es buscar que el estudiante permanezca en la carrera.

Debido a su lentitud de avance según el plan de estudio, se recomienda que busque completar bloques de cursos para que supere etapas. Adicionalmente, los estudiantes que se reintegran a sus estudios después de un período de suspensión, generalmente lo hacen en forma paulatina. Este tipo de estudiantes podría tener incluso que repetir cursos por la obsolescencia de su contenido, por lo cual es indicado verificar el cumplimiento de bloques.

Por otro lado, la excelencia académica sigue siendo deseable en el desempeño estudiantil y es parte de los intereses de la Universidad, por lo que permanece en el modelo.

La comparación pareada de estos objetivos mostró un índice de inconsistencia de 0.02. 


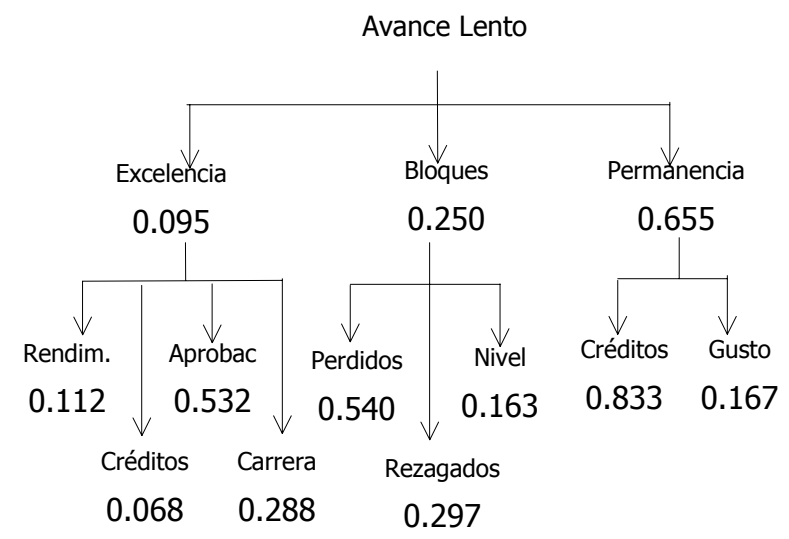

Figura 5: Modelo de avance lento

\subsubsection{Criterios}

Objetivo de permanencia en la carrera (con un índice de inconsistencia de 0.00):

1. Número de créditos mínimo: corresponde a la cantidad de créditos de un plan de matrícula, la cual se espera que sea baja. Cuanto menos créditos tenga un plan, mayor es su calificación. Este criterio es el primero en importancia ya que generalmente el principal problema de los estudiantes que avanzan lento es la falta de tiempo para los estudios, lo que les impide llevar suficientes créditos.

2. Gusto por los cursos: este criterio es muy importante dada la alta probabilidad de que un estudiante con poca disponibilidad de tiempo para el estudio abandone la carrera. Este criterio le da mayor puntaje al plan que motive más al estudiante, con el objetivo de evitar la deserción.

Objetivo de completar bloques (con un índice de inconsistencia de 0.01):

1. Porcentaje de cursos perdidos.

2. Porcentaje de cursos rezagados.

3. Porcentaje de cursos del nivel.

Estos criterios ya fueron explicados en el objetivo de completar bloques del modelo de excelencia académica. 
Objetivo de excelencia académica en la carrera (con un índice de inconsistencia de 0.01):

1. Probabilidad de aprobar todos los cursos: es el criterio más importante, ya que es indispensable que los pocos créditos que matricula el estudiante los apruebe, para no retrasar más su avance.

2. Cursos de la carrera: el llevarlos ayuda a minimizar el tiempo de culminación del plan de estudio.

3. Estimación del rendimiento académico: un buen rendimiento académico siempre es deseable en cualquier estudiante.

4. Número de créditos: un número elevado de créditos ocupa el último lugar de prioridad, dadas las limitaciones del estudiante en este sentido.

La organización jerárquica de estos objetivos y criterios se muestra en la figura 5.

\subsubsection{Función objetivo:}

$0.655^{*}\left(0.833^{*}\right.$ Créd $+0.167^{*}$ Gusto $)+$

$0.250 *\left(0.540 *\right.$ Perd $+0.297^{*}$ Rezag $+0.163 *$ Nivela $)+$

$0.095^{*}\left(0.112^{*}\right.$ Rend $+0.068^{*}$ Créd $+0.532^{*}$ Aprob $+0.288^{*}$ Carr $)$

\subsection{Modelo de bajo rendimiento (figura 6)}

El objetivo principal de este modelo es seleccionar el mejor plan de matrícula que ayude al estudiante en condición académica de atención especial o con matrícula restringida a obtener un rendimiento académico aceptable (mayor o igual a 7.0).

\subsubsection{Objetivos}

El objetivo más importante en este tipo de estudiante es recuperar su rendimiento, ya que un promedio bajo le afecta en su solicitud de matrícula y en becas, entre otras cosas. También se debe tratar de motivarlo para que se le facilite el proceso de recuperación. Las 
comparaciones pareadas de estos dos objetivos arrojaron un índice de inconsistencia de 0.00 .

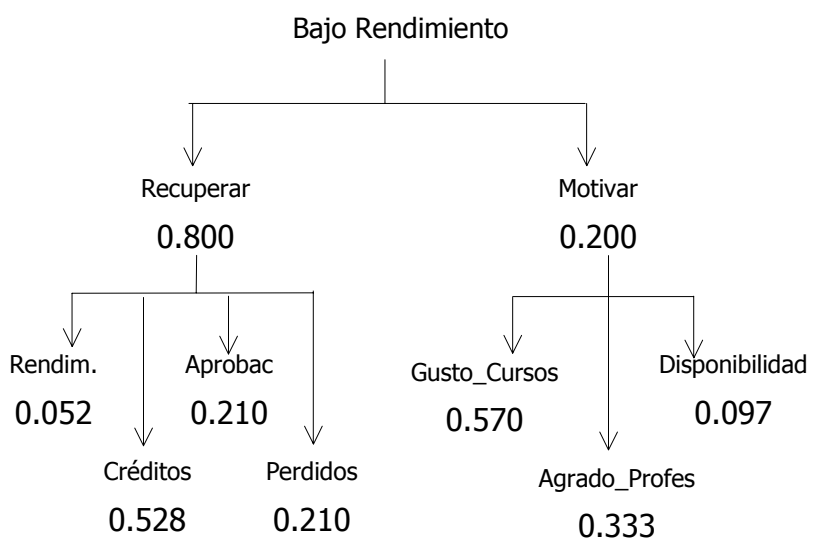

Figura 6: Modelo de bajo rendimiento

\subsubsection{Criterios}

Objetivo de recuperar rendimiento (con un índice de inconsistencia de 0.03 ):

1. Número de créditos máximo: es la cantidad de créditos que incluye el plan de matrícula, la cual será limitada a un máximo de acuerdo con el criterio del profesor y la reglamentación existente. Entre menos créditos tenga el plan, mayor calificación obtiene en este criterio. El objetivo de la limitación es que el estudiante concentre sus esfuerzos en pocos créditos para que recupere su promedio.

2. Probabilidad de aprobar todos los cursos: este criterio es muy importante, ya que de esto dependerá el que obtenga un promedio mayor o igual a 7.

3. Porcentaje de cursos perdidos: el estudiante debe superar lo antes posible los obstáculos que le dificultan mejorar su promedio ponderado, entre ellos, debe aprobar los cursos que haya perdido anteriormente.

4. Estimación del rendimiento académico: es el posible rendimiento que obtendrá el estudiante en el semestre siguiente. A pesar de que el objetivo fundamental es que mejore 
su condición a un promedio por lo menos de 7.0 , sería muy positivo si además obtiene un rendimiento académico alto.

Objetivo de motivar (con un índice de inconsistencia de 0.02):

1. Gusto por los cursos: la motivación principal está en el gusto que el estudiante desarrolle por los contenidos temáticos de los cursos. Este criterio le da mayor puntaje al plan de matrícula que motive más al estudiante, con el objetivo de evitar la deserción en la carrera.

2. Agrado por los profesores: los profesores de los cursos influyen en la actitud que el estudiante desarrolle hacia los cursos, por lo que para el estudiante de bajo rendimiento es importante que se identifique con sus profesores.

3. Disponibilidad de los profesores: los estudiantes de bajo rendimiento pueden verse beneficiados si los profesores les brindan una atención especializada en los temas de los cursos, por ejemplo, mediante un plan de acción individual (UCR, 2001). Este criterio le da mayor puntaje al plan de matrícula cuyo grupo de profesores tenga mayor disponibilidad de brindarle atención académica especial al estudiante.

En este modelo es importante tomar en cuenta la información de las razones del bajo rendimiento del estudiante, por ejemplo: historia académica, presencia de dificultades específicas para el aprendizaje, necesidad de apoyo profesional en el campo psicológico u otro. De esta forma el modelo podría ser ajustado a las necesidades de cada estudiante.

La jerarquía que organiza todos estos objetivos y criterios se muestra en la figura 6.

\subsubsection{Función objetivo:}

$0.800 *\left(0.052^{*}\right.$ Rend $+0.528^{*}$ Créd $+0.210^{*}$ Aprob + 0.210*Perd $)+$

$0.200 *\left(0.570^{*}\right.$ Gusto_Cursos $+0.333^{*}$ Agrado_Prof $+0.097^{*}$ Dispon $)$

Este modelo es otro ejemplo claro en donde la asignación de los pesos a los criterios depende fuertemente de la opinión y experiencia del tomador de decisiones que use el 
modelo. En este modelo de bajo rendimiento propuesto, tratando de tomar en cuenta el Reglamento de Régimen Académico Estudiantil en su definición de estudiantes con matrícula restringida (UCR, 2001, cap.1, art.3, inc. $r$ y y), se le dio mayor importancia a que el estudiante limite la cantidad de créditos a matricular. Sin embargo, podría considerarse que lo más importante es la posibilidad de aumentar el rendimiento académico, sobre todo en circunstancias que muestran que el estudiante ha venido mejorando su promedio ponderado paulatinamente.

\subsection{Modelo de formación académica amplia (figura 7)}

El objetivo principal de este modelo es seleccionar el plan de matrícula que mejor responda al deseo de adquirir conocimientos de diversos campos. También se considera importante que el estudiante mantenga un rendimiento académico alto.

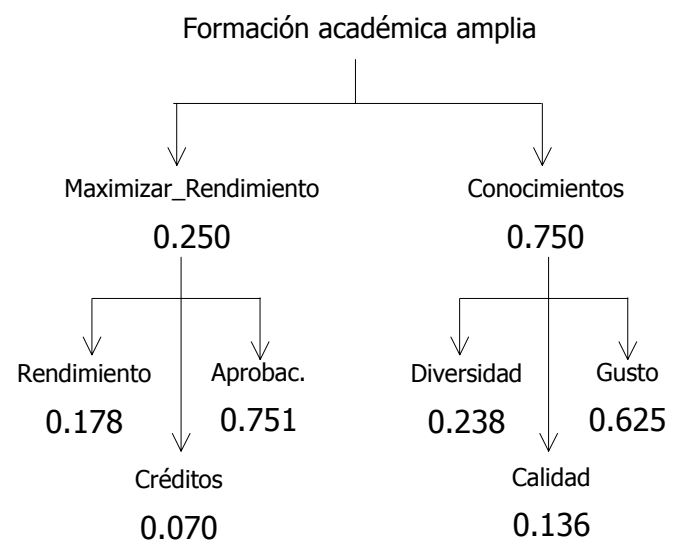

Figura 7: Modelo de formación académica amplia

\subsubsection{Objetivos}

El objetivo de ampliar conocimientos busca que el estudiante adquiera una variedad amplia de conocimientos de diversas áreas y que sean de su agrado. También se toma en cuenta como segundo objetivo el mantener un rendimiento académico alto. La comparación de estos objetivos tiene un índice de inconsistencia de 0.00 .

\subsubsection{Criterios}

Objetivo de ampliar conocimientos (con un índice de inconsistencia de 0.02): 
1. Gusto por los cursos: lo principal que motiva a los estudiantes de formación académica amplia es el gusto de matricular cursos de su interés. Por lo que este se convierte en el principal criterio del modelo.

2. Diversidad de cursos: un plan de matrícula con cursos de diferentes áreas de estudio obtiene una buena calificación en este criterio.

3. Calidad de cursos: este criterio mide la calidad promedio de los cursos del plan de matrícula, incluyendo contenido de los cursos, tipo de profesores, material de apoyo, etc. Es posible que la calidad del curso sea un criterio que este tipo de estudiantes toma en cuenta en el momento de matricular.

Objetivo de maximizar rendimiento (con un índice de inconsistencia de 0.03):

1. Probabilidad de aprobar todos los cursos: el criterio más importante es mantener un promedio al menos aceptable (mayor o igual a 7.0). Esto le facilita, además, encontrar cupo en los diversos cursos que desea matricular.

2. Estimación del rendimiento académico: otro objetivo deseable es que obtenga un buen rendimiento académico, lo que le favorece aún más en encontrar cupo en los cursos deseados. Esto cobra importancia en el modelo de formación académica amplia ya que los cursos a matricular podrían tener una demanda a nivel de toda la población universitaria y no sólo entre los estudiantes de la Unidad Académica.

3. Número de créditos: muchos de los estudiantes que pertenecen al perfil de formación académica amplia cursan varias carreras simultáneamente, por lo que su carga académica tiende a ser elevada. Se sugiere que un plan de matrícula con 16 o más créditos (estudiante de tiempo completo) obtenga la calificación máxima en este criterio.

La jerarquía de este modelo se muestra en la figura 7.

\subsubsection{Función objetivo:}

$0.250 *\left(0.178^{*}\right.$ Rend $+0.070^{*}$ Créd $+0.751^{*}$ Aprob $)+$ 


\section{$0.750 *\left(0.238^{*}\right.$ Diversidad $+0.136^{*}$ Calidad $+0.625^{*}$ Gusto $)$}

\section{Uso de los modelos}

En una situación real, el profesor consejero debe seleccionar el perfil que mejor represente al estudiante y utilizar el modelo respectivo. Como se dijo anteriormente, los pesos del modelo pueden modificarse para adaptarse a las prioridades y los objetivos del profesor y del estudiante. Incluso la jerarquía podría también variarse para incluir algún factor importante o excluir otro que no aplique al caso particular. En el caso que se realicen modificaciones al modelo original, la función objetivo se ajustará en consecuencia.

Luego, la función objetivo del modelo debe aplicarse a cada una de las alternativas de planes de matrícula que plantee ya sea el estudiante o el profesor como válidas. Esto da como resultado una calificación a cada uno de los planes, donde la calificación más alta corresponde al mejor plan.

Como ejemplo, supóngase que se realizará la orientación académica a un estudiante de bajo rendimiento que usualmente lleva en promedio 12 créditos y aprueba sólo 6 , y que tiene dos alternativas de matrícula: el plan A con 12 créditos y el plan B con 4 créditos. El plan A incluye 2 de los 3 cursos reprobados por el estudiante y el plan B incluye 1 de los 3 cursos reprobados. Una vez evaluadas éstas y el resto de características de cada plan, se utiliza la función objetivo correspondiente al perfil de bajo rendimiento y se aplica a los dos planes de matrícula, obteniéndose los siguientes resultados (utilizando una escala de 0 a 10 para evaluar los criterios o variables):

Plan $\mathbf{A}=$

$$
\begin{aligned}
& 0.8 *(0.528 * 5+0.210 * 6+0.210 * 6.66+0.052 * 5.99)+ \\
& 0.2 *(0.570 * 7+0.333 * 6+0.097 * 9)=5.86
\end{aligned}
$$

\section{Plan $\mathbf{B}=$}

$0.8 *(0.528 * 10+0.210 * 9+0.210 * 3.33+0.052 * 8.12)+$

$0.2 *(0.570 * 3+0.333 * 5+0.097 * 7)=7.44$ 
Se puede concluir con este resultado, dado en una escala de 0 a 10 , que el plan más adecuado para ayudar al estudiante a superar su bajo rendimiento según el modelo es el plan $B$, dado que obtuvo una calificación más alta que el plan $A$.

Como último paso de la orientación académica, se lleva a cabo la toma de decisión final, correspondiente al proceso 7 de la figura 1. Vale la pena destacar que en esta etapa pueden considerarse situaciones particulares que en realidad no corresponden a los perfiles definidos. Por ejemplo, restricciones en los horarios de los cursos es un factor que puede descartar la selección de un plan en la toma de decisión final.

\section{Conclusiones}

El enfoque dado a la orientación académica en este artículo, en donde se presenta como un sistema de procesos de análisis y toma de decisiones, favorece la posibilidad de mejorar la calidad de atención al estudiante. Permite el estudio de cada proceso con el objetivo de definir directrices que faciliten su ejecución y, de ser posible, desarrollar herramientas que apoyen a los tomadores de decisiones.

De esta forma es como se identificó que el utilizar perfiles estudiantiles regula y facilita el desempeño del profesor consejero, permitiéndole ser consistente en sus decisiones y a la vez brindar una atención individualizada mejorada, al tener como punto de partida en el análisis caracterizaciones estudiantiles predefinidas.

El enfoque de objetivos múltiples dado al problema condujo a modelos que permiten combinar las distintas necesidades de los dos tomadores de decisiones sin imponer un único criterio.

Además, la utilización del método AHP para diseñar y desarrollar los modelos propuestos, dio como resultado modelos que se acoplan a la naturaleza humana de resolución de conflictos y no fuerzan al tomador de decisiones a un modo de pensamiento que podría violar su mejor entendimiento de la situación. Es decir, los modelos son flexibles y permiten conciliar diversidad de objetivos y se ajustan fácilmente al estilo de toma de decisiones del usuario. 
A eso se suma que los modelos son capaces de adaptarse a los diferentes tipos de criterios de los profesores y a las necesidades específicas de los estudiantes, mediante la modificación de la jerarquía, pesos, escalas de evaluación y calificación de los factores o variables.

Dado el alto índice de variabilidad y subjetividad del problema abordado, la implementación computacional de los modelos propuestos requiere de un sistema versátil capaz de adaptarse a diferentes situaciones y que permita la intervención del usuario en el proceso de decisión. Esta capacidad es brindada por los sistemas de soporte a la toma de decisiones (DSS), los cuales se sugieren como herramienta de implementación de los modelos.

Finalmente, los modelos aquí descritos son resultado de un esfuerzo por brindar una solución de apoyo a un problema no estructurado como es la evaluación de diferentes planes de matrícula. Constituyen una propuesta inicial que pretende ser objeto de análisis y punto de partida de estudios posteriores, tendientes a mejorarla o a apoyar otros procesos de la orientación académica no abordados en este artículo, como por ejemplo el sistema de expedientes estudiantiles.

\section{Agradecimientos}

Agradecemos los aportes del Dr. Ronald Argüello V. y de la Dra. Gabriela Marín R., lectores de la tesis de maestría en Computación e Informática de la UCR, dentro de la cual se enmarca este artículo. Agradecemos también a la M.Sc. Patricia Ruh, jefe del Centro de Orientación Vocacional de la Oficina de Orientación de la UCR y a la psicóloga del Centro de Asesoría Estudiantil de la UCR, María Elena Rodríguez por la evaluación de este artículo.

\section{Referencias}

Biasetti, L.; Gutiérrez, L. y Quirós, T. (1991). Reconceptualización del Rol del Profesor Consejero y Normativa de Evaluación. San José, Costa Rica: Vicerrectoría de Vida Estudiantil, Universidad de Costa Rica.

Department of Computing, Imperial College of Science, Technology and Medicine, University of London (1996). Free On-Line Dictionary of Computing. Recuperado en 2002, de http://wombat.doc.ic.ac.uk/foldoc/foldoc.cgi?eigenvector. 
Fandel, G. y Spronk, J. (1985). Multiple Criteria Decision Methods and Applications. Berlín, Alemania: Springer-Verlag Heidelberg.

Forman, E. (1990). Multi criteria decision making and the analytic hierarchy process. Berlín, Alemania: Readings in Multiple Criteria Decision Aid, editor Carlos A. Bana e Costa.

Mazur, G. Manual Approximation of AHP. Recuperado en 1998, de http://wwwpersonal.engin.umich.edu/ gmazur/tam/ahpman.htm.

Murillo, M. y Lara, V. (2002). Modelo Jerárquico de Estimación del Rendimiento Académico Relativo Estudiantil utilizando el Método Analytic Hierarchy Process. San José, Costa Rica: Primer Congreso Nacional de Educación.

Olson, D. y Courtney, J. (1992). Decision Support Models and Expert Systems. New York, Estados Unidos: Macmillan Publishing Company.

Saaty, T. Presentación del libro: Decision Making for Leaders, AHP Series, Vol. II. Recuperado en 1996 de http://ahp.net/support/books/ books.htm.

Senn, J. (1987). Information Systems in Management. Tercera edición. Belmont, California, Estados Unidos: Wadsworth Publishing Company.

Universidad de Costa Rica. (1983). Reglamento de los Actos de Graduación. San Pedro, Costa Rica: La Gaceta Universitaria 06-83.

. (1987). Reglamento de Adjudicación de Becas y Otros Beneficios a los Estudiantes. San Pedro, Costa Rica: Centro de Información.

(2001). Reglamento de Régimen Académico Estudiantil. San Pedro, Costa Rica: La Gaceta Universitaria, No. 3-2001.

Universidad de Costa Rica, Oficina de Planificación Universitaria. (1996). Plan Operativo 1997. San José, Costa Rica.

Universidad de Costa Rica, Rectoría, Oficina de Planificación Universitaria. (1985). Plan Quinquenal de Desarrollo de la Universidad de Costa Rica 1986-1990. San José, Costa Rica: Oficina de Publicaciones de la Universidad de Costa Rica.

Universidad de Costa Rica, Vicerrectoría de Vida Estudiantil, Oficina de Registro e Información (2002). Instructivo para el Estudio de Graduación de Honor. San José, Costa Rica. 